\title{
The Pharmacokinetics of Caffeine in Preterm Newborns: No Influence of Doxapram but Important Maturation with Age
}

\author{
Aline G.J. Engbers ${ }^{a, b}$ Swantje Völler ${ }^{a, c}$ Christian F. Poets ${ }^{d}$ \\ Catherijne A.J. Knibbe $^{a, e}$ Irwin K.M. Reiss ${ }^{b}$ Birgit C.P. Koch ${ }^{f}$ Robert B. Flint $^{b, f}$ \\ Sinno H.P. Simons ${ }^{b}$ \\ aDivision of Systems Biomedicine and Pharmacology, LACDR, Leiden University, Leiden, The Netherlands; \\ ${ }^{b}$ Division of Neonatology, Department of Paediatrics, Erasmus UMC - Sophia Children's Hospital, Rotterdam, \\ The Netherlands; ' Division of BioTherapeutics, LACDR, Leiden University, Leiden, The Netherlands; ${ }^{\mathrm{d} D e p a r t m e n t}$ of \\ Neonatology, Tübingen University Hospital, Tübingen, Germany; ${ }^{~}$ Department of Clinical Pharmacy, St. Antonius \\ Hospital, Nieuwegein, The Netherlands; fDepartment of Hospital Pharmacy, Erasmus University Medical Center, \\ Rotterdam, The Netherlands
}

\section{Keywords}

Apnea · Caffeine · Doxapram · Pharmacokinetics · Drug therapy $\cdot$ Interaction

\section{Abstract \\ Background: Apnea of prematurity can persist despite caf- feine therapy in preterm infants. Doxapram may addition- ally support breathing. Although multiple small studies have reported the efficacy of doxapram, the structural co-treat- ment with caffeine impedes to ascribe the efficacy to doxa- pram itself or to a pharmacokinetic (PK) interaction where doxapram increases the exposure to caffeine. We examined whether there is a PK drug-drug interaction between doxa- pram and caffeine by developing a PK model for caffeine in- cluding infants with and without doxapram treatment. Methods: In preterm neonates receiving caffeine, we deter- mined caffeine plasma concentrations before, during, and directly after doxapram co-treatment and used these to de- velop a population PK model in NONMEM 7.3. Patient char-}

acteristics and concomitant doxapram administration were tested as covariates. Results: 166 plasma samples were collected from 39 preterm neonates receiving caffeine (median gestational age 25.6 [range 24.0-28.0] weeks) of which 65 samples were taken during co-treatment with doxapram (39\%, from 32/39 infants). Clearance of caffeine was 9.99 $\mathrm{mL} / \mathrm{h}$ for a typical preterm neonate with a birth weight of 0.8 $\mathrm{kg}$ and 23 days postnatal age and increased with birth weight and postnatal age, resulting in a 4-fold increase in clearance during the first month of life. No PK interaction between caffeine and doxapram was identified. Discussion: Caffeine clearance is not affected by concomitant doxapram therapy but shows a rapid maturation with postnatal age. As current guidelines do not adjust the caffeine dose with postnatal age, decreased exposure to caffeine might partly explain the need for doxapram therapy after the first week of life.

(C) 2021 The Author(s)

Published by S. Karger AG, Basel karger@karger.com www.karger.com/neo

Karger $\stackrel{\text { ' }}{5}$

BOPEN ACCESS
(C) 2021 The Author(s)

Published by S. Karger AG, Basel

This is an Open Access article licensed under the Creative Commons Attribution-NonCommercial-4.0 International License (CC BY-NC) (http://www.karger.com/Services/OpenAccessLicense), applicable to the online version of the article only. Usage and distribution for commercial purposes requires written permission.
Aline G.J. Engbers

Division of Systems Biomedicine and Pharmacology

LACDR, Leiden University, Einsteinweg 55

NL-2333 CC Leiden (The Netherlands)

a.g.j.engbers@lacdr.leidenuniv.nl 


\section{Introduction}

Neurological and respiratory immaturity in preterm infants is thought to contribute to apnea of prematurity. Apnea-related hypoxic episodes are harmful to the infant's neurodevelopment [1]. Caffeine is standard of care to support breathing after preterm birth, due to its beneficial effect on short- and long-term outcome [2,3]. Caffeine reduces the number of apneic events and the duration of mechanical ventilation $[2,4]$.

If apnea persists despite optimal noninvasive ventilatory support and caffeine treatment, coadministration of doxapram may be considered. This off-label respiratory stimulant has been associated with a reduced rate of hypoxic events and duration of mechanical ventilation as well as less bronchopulmonary dysplasia [5-8]. A reduction in oxygen requirements has also been shown but is limited by the small number of studies [7-10]. Ten Hove et al. suggested that doxapram is associated with an improved neurodevelopmental outcome at the age of 2 years [11].

The limited evidence on doxapram efficacy, together with the structural co-treatment with caffeine, calls for an investigation whether the observed effects upon start of doxapram treatment can be attributed to doxapram itself. In the past, when therapeutic drug monitoring caffeine was still part of routine care, occasionally very high caffeine concentrations were observed when doxapram therapy was coadministered (unpublished data). Before doxapram can become a structural part of the apnea of prematurity treatment plan, it is essential to exclude the existence of a pharmacokinetic $(\mathrm{PK})$ interaction between caffeine and doxapram. Therefore, the current study aimed to investigate if doxapram co-treatment affects caffeine PKs in preterm infants as a result of a PK interaction. To this end, a population PK modeling approach was applied, as this approach allows for the analysis of clinically obtained (sparse) data and can provide knowledge on the PK of a drug in clinical practice $[12,13]$. For our research question, caffeine concentration data from individuals before, during, or after co-treatment with doxapram together with data from individuals that received caffeine treatment only, that were all obtained during opportunistic sampling as part of the DINO study, and were analyzed together. The results were used to develop a population $\mathrm{PK}$ model for caffeine in preterm infants to examine the existence of a 1-way drug-drug interaction between doxapram use and caffeine PKs. Additionally, the population PK model was used to evaluate the exposure to caffeine upon the use of different dosing strategies.

No PK Interaction between Caffeine and Doxapram

\section{Methods}

\section{Patients and Treatment}

Patients were admitted at the neonatal intensive care unit of Erasmus Medical Centre and were enrolled in the Drug dosage Improvement in NeOnates (DINO) study, in which preterm neonates born before 32 weeks of gestation were included. The local Ethics Committee approved the protocol, and written informed consent from parents/legal guardians was obtained (MEC-2014-067, NCT02421068). Patients were treated with caffeine (as base, $10 \mathrm{mg}$ / $\mathrm{mL}$, Pharmacy A15, Gorinchem, The Netherlands) starting with a loading dose of $10 \mathrm{mg} / \mathrm{kg}$, followed by 1 daily maintenance dose of $5 \mathrm{mg} / \mathrm{kg}$. Additional caffeine doses were allowed, and the maintenance dose could be increased up to $8 \mathrm{mg} / \mathrm{kg}$. Doxapram administration (as hydrochloride, Dopram ${ }^{\circledR}$, Eumedica, Manage, Belgium) was initiated if the attending physician judged that apnea or bradycardia persisted despite caffeine therapy and noninvasive ventilatory support. A doxapram loading dose of $2.5 \mathrm{mg} / \mathrm{kg}$ was administered intravenously over $15 \mathrm{~min}$ at discretion of the attending physician, followed by a maintenance dose of $2.0 \mathrm{mg} / \mathrm{kg} / \mathrm{h}$ via continuous intravenous infusion or gastro enteral administration. In case of clinical improvement, the doxapram dose could be decreased stepwise. Doxapram was stopped upon need for endotracheal intubation or upon disappearance of apnea, regarded as treatment success. Both caffeine and doxapram were allowed to be switched to gastro enteral administration once enteral feeds were well tolerated.

\section{Blood Samples}

Blood samples of $0.2 \mathrm{~mL}$ were collected in EDTA-tubes and withdrawn from an indwelling arterial catheter or with routinely scheduled samples for clinical purposes. Samples were collected during caffeine and caffeine plus doxapram treatment period. Opportunistic sampling did not exceed $1 \%$ of the total blood volume per day and $3 \%$ of the total blood volume per 4 weeks. Directly after collection, samples were stored at $2-8^{\circ} \mathrm{C}$. Within $24 \mathrm{~h}$, the sample was centrifuged, and plasma was stored at $-80^{\circ} \mathrm{C}$ until quantification.

\section{Bioanalytical Analysis}

Caffeine plasma concentrations were measured using ultraperformance liquid chromatography-electrospray ionization-tandem mass spectrometry (UPLC-ESI-MS/MS) at the Pharmacy Department of the Erasmus Medical Centre, Rotterdam, The Netherlands. The assay was validated according to FDA guidelines, required $50 \mu \mathrm{L}$ plasma volume, and was linear over a caffeine concentration range of $0.75-50 \mathrm{mg} / \mathrm{L}$ (coefficient of variation intraassay: $2.4 \%$, coefficient of variation inter-assay: $3.5 \%$ ). The lower limit of the range represents the lower limits of quantification, and the limit of detection was $0.46 \mathrm{mg} / \mathrm{L}$. Concentrations below the lower limit of quantification ( $0 \%$ of caffeine concentrations) and above the upper limit of quantification (4\%) were reported by the laboratory and were used in the analysis.

Model Development

A population PK model was developed in NONMEM V7.3 (ICON Development Solutions, Ellicott City, MD, USA). R version 3.5.1 was used in R-studio version 1.1.463 to build the dataset and visualize the data and model output.

Population PK model development was based on change in objective function value (dOFV), evaluation of goodness of fit (ob- 
Table 1. Patient characteristics

\begin{tabular}{lc}
\hline & Median (range) \\
\hline Patient characteristics & $39[62]$ \\
patients, $n$ [\%male] & $800(485-1,290)$ \\
Birth weight, g & $25.6(24.0-28.0)$ \\
Gestational age, weeks & $9(23 \%)$ \\
Small for gestational age & $23(0-61)$ \\
Postnatal age during caffeine treatment, days* & $990(450-2,160)$ \\
Current body weight during caffeine treatment, g* & $32(82)$ \\
Patients receiving doxapram, $n$ (\%) & $21(5-40)$ \\
Postnatal age at doxapram initiation, days* & $32(5-61)$ \\
Postnatal age during doxapram treatment, days* & $1,160(700-2,160)$ \\
Current body weight during doxapram treatment, g* & $38(3-61)$ \\
\hline Caffeine and doxapram treatment and dosing & $10(5-11)[100]$ \\
Caffeine treatment duration, days & $5(3-10)$ \\
Caffeine loading dose, mg/kg [\% I.V. doses] & $35(1-60)[41]$ \\
Caffeine maintenance dose, $\mathrm{mg} / \mathrm{kg} / 24$ h* & $5(3-9)$ \\
Caffeine maintenance doses per patient during study [\% I.V. doses], $n$ & $5(1-52)[45]$ \\
Additional caffeine doses, mg/kg & \\
Additional caffeine doses per patient $(n)[\%$ I.V. doses], $n$ & $14(1-49)$ \\
\hline Doxapram & $7(2-23)[46]$ \\
Doxapram treatment duration, days & $349(25-1,463)$ \\
Doxapram administrations per patient $(n)$ [\% I.V. doses], $n$ & $22.8(1.0-68.6)$ \\
Total doxapram dose per patient, mg/kg & $2.4(1.0-2.7)[81]$ \\
Doxapram dose per patient per day, mg/kg/day* & $2.0(0.9-2.9)[71]$ \\
First doxapram loading dose, mg/kg/15 min [\% I.V. doses] & \\
First doxapram maintenance dose, mg/kg/h [\% I.V. doses] & \\
\hline
\end{tabular}

* Of time-changing characteristics, the median numbers of individual median throughout the treatment period are presented.

served concentrations vs. population and individual predicted concentrations, conditional weighted residuals vs. population predicted concentration and time after last caffeine dose) plots, and numerical performance (relative standard error below 30\%). Due to limited plasma caffeine samples in the absorption phase during oral administration, we fixed the absorption rate to $4.0 \mathrm{~h}^{-1}$ and bioavailability to $100 \%$ [14].

A covariate analysis was performed testing the available patient characteristics and doxapram use as potential covariates. Birth weight, current weight, postnatal age, gestational age, gender, and small for gestational age were plotted against inter-individual variability (IIV) of clearance and volume of distribution to select covariates to test on the structural model. The effect of doxapram therapy was tested on caffeine clearance as doxapram naivety (clearance before doxapram therapy vs. clearance during- and after-doxapram administration) and as concomitant doxapram therapy (clearance before- vs. during- vs. after-doxapram administration). To prevent missing an interaction as a result of a slower offset effect of doxapram on caffeine CL, samples taken up to 24, 48 , or $72 \mathrm{~h}$ after stopping doxapram administration were included in the during-doxapram administration group in a sensitivity analysis. Covariates were included if dOFV was $<-6.6(p<0.01)$. A backward elimination procedure with a minimal dOFV of 10.8 $(p<0.001)$ was initiated when inclusion of covariates did not further improve the model. The final model was validated by performing a bootstrap, a normalized prediction distribution error (NPDE) analysis and a prediction corrected visual predictive check, each based on 1,000 simulations.

\section{Dose Evaluation}

Based on the final model that was developed in this study, exposure to caffeine was illustrated for a median preterm infant with a GA of 26 weeks and a birth weight of $0.83 \mathrm{~kg}$. Postnatal weight loss and gain were based on predictions by https://www.growthcalculator.org/. According to clinical practice, during the first 14 days of life, the caffeine dose was based on birth weight, and above 14 days, the absolute dose was based on current weight. Three different dosing regimens were evaluated, all starting with a loading dose of $10 \mathrm{mg} / \mathrm{kg}$ caffeine base. The maintenance dosages were either $2.5 \mathrm{mg} / \mathrm{kg} /$ day and $5 \mathrm{mg} / \mathrm{kg} /$ day or as proposed by Koch et al. [15], that is, $2.5 \mathrm{mg} / \mathrm{kg} /$ day during the first week, $3 \mathrm{mg} / \mathrm{kg} /$ day during the second week, $3.5 \mathrm{mg} / \mathrm{kg} /$ day during weeks 3 and 4 , and $4 \mathrm{mg} / \mathrm{kg} /$ day from week 5 to 8 [15]. Median caffeine concentrations were calculated from 1,000 simulations. 


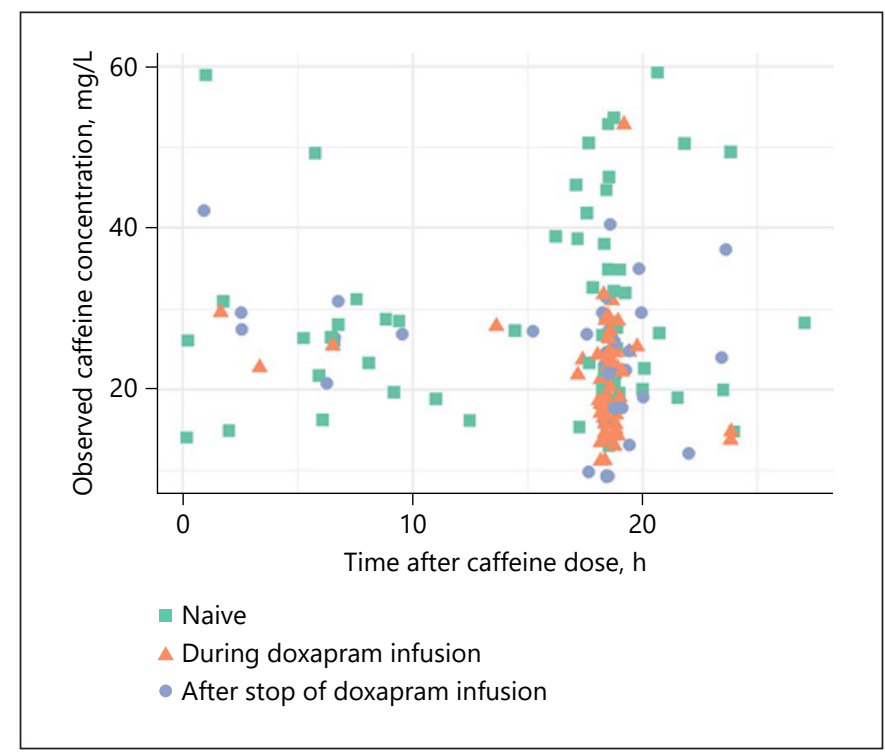

Fig. 1. Observed caffeine plasma concentrations versus time after last dose.

\section{Results}

\section{Patients and Samples}

In 39 patients, 166 plasma caffeine samples were collected. Doxapram therapy was initiated in 32 patients during the study period. Sixty-three samples (38\%) were taken before doxapram therapy was initiated, 65 (39\%) during-doxapram administration, and 38 (23\%) samples after stopping doxapram. Of the latter 38 samples, 10 samples were taken within $24 \mathrm{~h}$ after stopping doxapram administration, 11 within $24-48 \mathrm{~h}$, and 2 (1\%) within 48$72 \mathrm{~h}$ after stopping doxapram. Patient characteristics as well as dosing information are presented in Table 1. Observed caffeine plasma concentrations are presented in Figure 1.

\section{Population Pharmacokinetic Model}

A one-compartment model with a proportional residual error model described the data best. IIV was identified on CL but could not be identified on volume of distribution $(\mathrm{V})$. Addition of a power-relationship between clearance and PNA to the base model resulted in a dOFV of $-134.0(p<0.001)$ and explained $8.3 \%$ of the IIV on CL. Addition of a power-relationship between clearance and birth weight further decreased the OFV with 15.5 points $(p<0.001)$ and explained another $5 \%$ of the IIV on CL.

Comparison of doxapram naïve versus doxapram treated caffeine clearance suggested a 17\% higher caffeine
Table 2. Parameter and bootstrap estimates of the final population PK model

\begin{tabular}{|c|c|c|}
\hline & $\begin{array}{l}\text { Final model } \\
\text { estimate (RSE \%) } \\
\text { [shrinkage \%] }\end{array}$ & $\begin{array}{l}\text { Bootstrap estimate } \\
(95 \% \text { CI })\end{array}$ \\
\hline \multicolumn{3}{|c|}{$C L_{\text {ind }}=C L_{\text {pop }} \times\left(P N A_{\text {ind }} / 23 \text { days }\right)^{\Theta P N A} \times\left(W T_{B, \text { ind }} / 0.8 \mathrm{~kg}\right)^{\Theta W T} B$} \\
\hline $\mathrm{CL}_{\text {pop }}, \mathrm{mL} / \mathrm{h}$ & $9.99(4)$ & $9.93(9.20-10.7)$ \\
\hline$\Theta P N A$ & $0.505(16)$ & $0.519(0.401-0.815)$ \\
\hline$\Theta W T B$ & $0.707(20)$ & $0.732(0.438-1.049)$ \\
\hline \multicolumn{3}{|l|}{$V_{\text {ind }}=V_{\text {pop }}$} \\
\hline \multicolumn{3}{|c|}{ Inter-individual variability } \\
\hline IIV on clearance, $\%$ & $20.5(11)[11]$ & $19.6(14.9-23.9)$ \\
\hline \multicolumn{3}{|c|}{ Residual unexplained variability } \\
\hline Proportional error & $0.0462(20)[9]$ & $\begin{array}{l}0.0435(0.0246- \\
0.0628)\end{array}$ \\
\hline
\end{tabular}

$\mathrm{CL}_{\text {ind }}$, individual caffeine clearance in $\mathrm{mL} / \mathrm{h} ; \mathrm{CL}_{\text {pop }}$, population caffeine clearance in $\mathrm{mL} / \mathrm{h}$ for a typical individual with a postnatal age of 23 days and a birth weight of $0.8 \mathrm{~kg}$; $\mathrm{PNA}_{\text {ind }}$, individual postnatal age in days; $\mathrm{WT}_{\mathrm{B} \text {,ind }}$, individual birth weight in $\mathrm{kg} ; V_{\text {ind }}$, individual volume of distribution in $\mathrm{mL} ; V_{\text {pop }}$, population volume of distribution in $\mathrm{mL}$ for a typical individual with a postnatal age of 23 days and a birth weight of $0.8 \mathrm{~kg}$; IIV, inter-individual variability; PK, pharmacokinetic; RSE, relative standard error.

clearance in doxapram treated patients, but this effect did not meet the significance and precision criteria (dOFV $-3.5[p>0.05]$ and relative standard error 99\%). In the sensitivity analysis, dOFV ranged from -4.7 when concomitant doxapram use was defined for samples taken during-doxapram infusion to -3.6 when samples taken up to $72 \mathrm{~h}$ after stopping doxapram infusion were considered as concomitant doxapram use as well $(p>0.05)$. Based on these results, doxapram therapy was not included as a covariate and did not significantly affect the clearance of caffeine.

As no other significant covariates were identified, backward elimination analysis was performed in which both covariates, that is, birth weight and PNA on clearance, remained significant $(\mathrm{dOFV}+15$ and +145 , respectively). Final model parameters and bootstrap estimates are presented in Table 2. The estimated maturation of clearance is visualized in Figure 2 and compared with previously reported maturation profiles in Figure 3. Goodness of fit plots of the final model is presented in (see online suppl. Fig. 1, 2; for all online suppl. material, see www. karger.com/doi/10.1159/000513413), remaining IIV on $\mathrm{CL}$ is presented in online suppl. Fig. 3, results of the nor- 


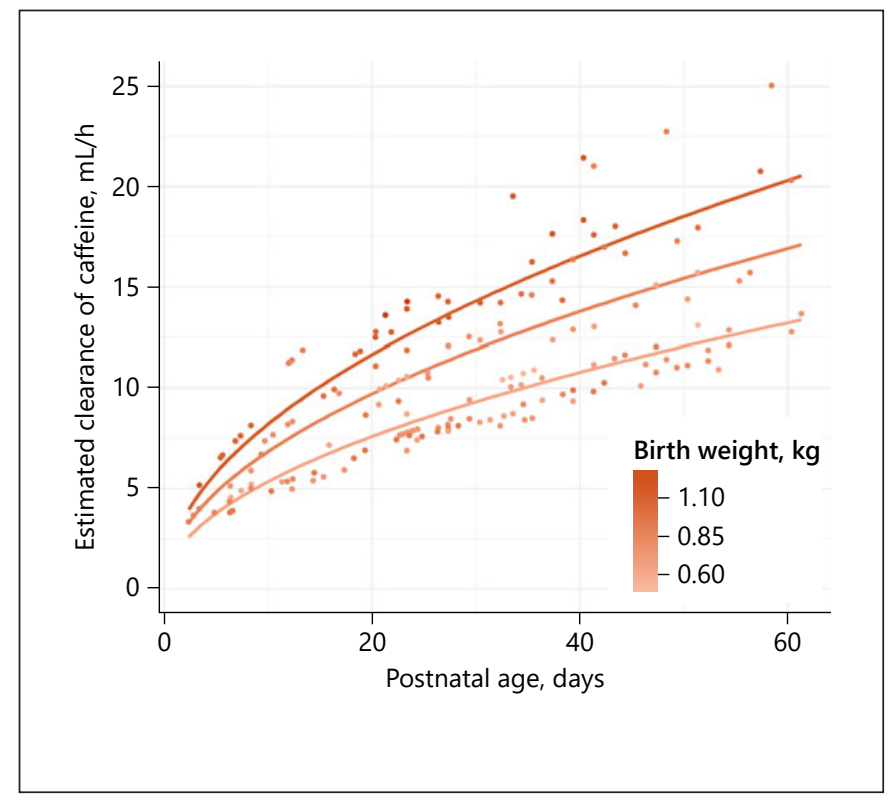

Fig. 2. Predicted maturation of caffeine clearance (lines) for preterm neonates with a birth weight of 0.60 (light orange), 0.85 (orange), or $1.10 \mathrm{~kg}$ (dark orange). Individual post hoc clearance estimates are presented as dots, with color intensity increasing with birth weight.

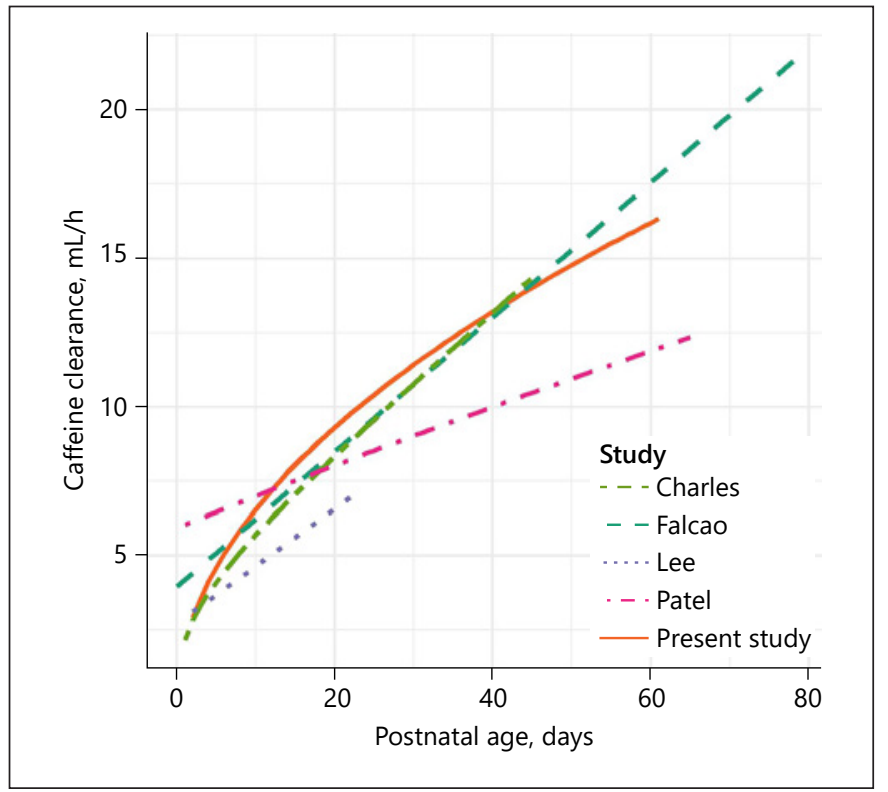

Fig. 3. Caffeine clearance as described in literature (14.23-25) (dashed lines). For the present study, population clearance versus postnatal age is given for the median birth weight of $0.8 \mathrm{~kg}$. For other studies, weight was predicted by a linear regression model based on observed weights in the study population. For each study, the predicted maturation is presented only for the range in postnatal age.

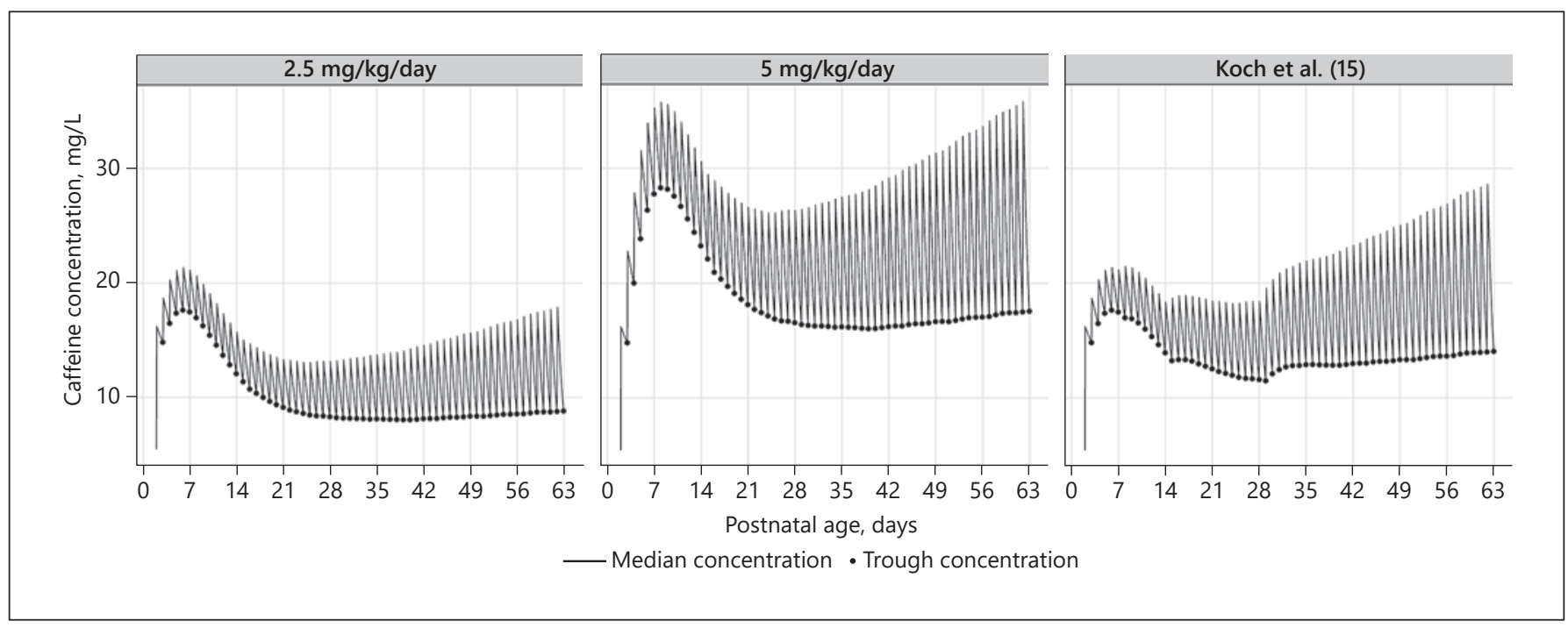

Fig. 4. Simulated caffeine concentrations in a median preterm infant with a gestational age of 26 weeks and birth weight of $0.83 \mathrm{~kg}$. In all 3 scenarios, a loading dose of $10 \mathrm{mg} / \mathrm{kg}$ was given. For the left scenario, a maintenance dose of $2.5 \mathrm{mg} / \mathrm{kg} /$ day was given, while for the middle scenario the maintenance dose was $5 \mathrm{mg} / \mathrm{kg} / \mathrm{day}$. For the right scenario, based on Koch et al. [15], the maintenance was
$2.5 \mathrm{mg} / \mathrm{kg} /$ day during the first week, $3 \mathrm{mg} / \mathrm{kg} /$ day during the second week, $3.5 \mathrm{mg} / \mathrm{kg} /$ day during the third and fourth week, and 4 $\mathrm{mg} / \mathrm{kg} /$ day from the fifth week and up. Solid lines represent the median concentration and dots represent a trough concentration, both based on 1,000 simulations. 
malized prediction distribution error analysis are presented in online suppl. Fig. 4, and a prediction corrected visual predictive check is presented in online suppl. Fig. 5.

\section{Dose Evaluation}

In Figure 4, the exposure to caffeine following different dosing strategies is presented. A constant maintenance dose of either 2.5 or $5 \mathrm{mg} / \mathrm{kg} /$ day results in relatively high trough concentrations during the first week of life (maximal concentration of 17.6 and $28.3 \mathrm{mg} / \mathrm{L}$, respectively). During the second and third week, the trough concentrations decrease until a minimum is reached during the fourth week ( 8.2 and $16.4 \mathrm{mg} / \mathrm{L}$, respectively), from where they remain stable. The maintenance dose adjustments to PNA, as suggested by Koch et al. [15], prevent the large differences between trough concentrations throughout the treatment period but still lead to a decrease in trough concentrations after the first week of life (from 17.6 in week 1 to $11.5 \mathrm{mg} / \mathrm{L}$ in week 4 ).

\section{Discussion}

We studied the PKs of caffeine in preterm newborns before, during, and after doxapram therapy, to investigate whether there is an interaction between doxapram and caffeine PK. In the current study, such a PK interaction was not identified.

For our study, we had access to data representative of the clinical setting that was collected without causing any burden to the patient. The population PK approach used in this study allowed the identification of the PK of caffeine based on sparse data and has proven its worth before for similar datasets of preterm infants [16, 17]. Concomitant doxapram therapy was a covariate of special interest due to its structural co-treatment to caffeine. The covariate analysis did not identify doxapram treatment naivety or concomitant doxapram treatment as significant predictor of caffeine clearance. Even when the concomitant doxapram therapy definition was extended to 24,48 , or $72 \mathrm{~h}$ after stopping doxapram, the effect remained insignificant, which excludes the presence of a PK interaction between doxapram and caffeine. A drug-drug interaction can also be of a pharmacodynamic (PD) nature. A synergistic PD interaction between caffeine and doxapram is not expected because they act via different mechanisms, but based on our study, this cannot be excluded. Recently, novel methods have been identified to quantify the amount of apnea using monitor data that might be useful for the investigation of a PD interaction between doxa-

No PK Interaction between Caffeine and Doxapram pram and caffeine $[18,19]$. Based on the present results, we can conclude that the previously reported effects of doxapram on apnea in preterm infants cannot be attributed to increased caffeine concentrations [5-7].

In our analysis, we observed an important maturation of caffeine clearance with PNA. Next to the important effect of PNA, we also found that clearance is predicted by birth weight which was independent of PNA, and for which, a cutoff value of PNA for the effect of birth weight could not be identified. The PK of caffeine has been described previously, with clearance of caffeine predicted by PNA and current body weight [14, 20-23]. In Figure 3, the reported increase in clearance with PNA is presented for the current and previous studies, which shows that the predicted effect of PNA on caffeine clearance is in line with previously reported maturation profiles [14, 20-23]. Figure 4, left and middle panel, shows how exposure to caffeine changes throughout the treatment period when the 2.5 or $5.0 \mathrm{mg} / \mathrm{kg}$ maintenance dose is not adjusted for PNA. The decreasing trough concentrations after the first week of treatment are a result of the rapid increase in CL. After the fourth week of treatment, a slight increase in trough concentrations is observed, which is likely to be an effect of the rapid increase in body weight but a less rapid increase in CL. In other words, body weight and, therefore, absolute dose increase more rapidly than CL at higher PNAs. From these results, we can even speculate that the lower caffeine concentrations in our population might have contributed to the decision to start concomitant doxapram therapy that was initiated at a median PNA of 21 days in our population. A stepwise increase in absolute dose with increasing PNA, as suggested by Koch et al. [15] (Fig. 4, right panel), results in a smaller difference in trough concentrations after the first week of life. Therefore, clinicians should be aware that patients might require a higher caffeine dose in $\mathrm{mg} / \mathrm{kg}$ after the first week of life.

The opportunistic sampling method allowed us to collect samples from a vulnerable population, with minimal burden for the patients. Because of this approach, most samples were obtained from routine blood sampling for clinical purposes, which was mostly scheduled around 8:00 a.m. As patients were scheduled to receive their caffeine maintenance dose at 2:00 p.m., most samples were taken around $18 \mathrm{~h}$ after the last caffeine dose (Fig. 1). Although our developed caffeine PK model was suitable to detect a potential interaction with doxapram, the small variation in time after dose limits the complete characterization of caffeine PK in preterm infants, especially for volume of distribution. Even though the population PK approach we used resulted in a population PK model 
whose estimated parameters are well in line with previously reported studies, caution is requested upon interpretation of the peak plasma concentrations presented in Figure 4. [14, 20-23]. Because we could not identify an influence of weight on V in our data, in Figure 4, the peak plasma concentrations increase with an increase in dose, which may not be realistic, and therefore, this model is less suitable to evaluate potential toxicity. Since CL was well identified in the model, the predicted trough concentrations are likely to be reflective of reality.

In our analysis, doxapram therapy was tested as a categorical covariate yes/no. As doxapram metabolism is reported to increase with PNA and GA [24], neonates may have been exposed to varying concentrations of doxapram. If our results would have suggested the presence of an interaction between doxapram and caffeine, doxapram concentrations would have provided a more precise investigation. Since we did not observe any signs for the presence of this interaction upon the maximum described doxapram dose administered, we do not expect that this interaction will arise when doxapram concentrations are studied in the model.

\section{Conclusion}

Caffeine clearance is not affected by doxapram therapy, but it does show a rapid maturation with postnatal age. The potential therapeutic effect of doxapram can, therefore, not be attributed to increased caffeine concentrations. The decreased exposure to caffeine upon increasing PNA might party explain the need for doxapram therapy after the first week of life.

\section{Statement of Ethics}

The Erasmus MC ethics review board approved the protocol and written informed consent from parents/legal guardians was obtained prior to study initiation (MEC-2014-067).

\section{Conflict of Interest Statement}

The authors have no conflicts of interest to declare.

\section{Funding Sources}

The DINO study was enabled by funding from The Netherlands Organization for Health Research and Development ZonMw (Grant No. 80-83600-98-10190).

\section{Author Contributions}

C.F.P., R.B.F., and S.H.P.S. conceived the presented idea; I.K.M.R. and S.H.P.S. collected the data, A.G.J.E., S.V., B.C.P.K., and C.A.J.K. performed the analysis; A.G.J.E., S.V., C.F.P., C.A.J.K., I.K.M.R., R.B.F., and S.H.P.S. interpreted the results. A.G.J.E. and S.H.P.S. wrote the manuscript with input from all authors.

\section{References}

1 Poets CF, Roberts RS, Schmidt B, Whyte RK, Asztalos EV, Bader D, et al. Association between intermittent hypoxemia or bradycardia and late death or disability in extremely preterm infants. JAMA. 2015;314(6):595-603.

2 Schmidt B, Roberts RS, Davis P, Doyle LW, Barrington KJ, Ohlsson A, et al. Caffeine therapy for apnea of prematurity. N Engl J Med. 2006;354(20):2112-21.

3 Schmidt B, Anderson PJ, Doyle LW, Dewey D, Grunau RE, Asztalos EV, et al. Survival without disability to age 5 years after neonatal caffeine therapy for apnea of prematurity. JAMA. 2012;307(3):275-82.

4 Kumar VHS, Lipshultz SE. Caffeine and clinical outcomes in premature neonates. Children. 2019;6(11).

5 Prins SA, Pans SJ, van Weissenbruch MM, Walther FJ, Simons SH. Doxapram use for apnoea of prematurity in neonatal intensive care. Int J Pediatr. 2013;2013:251047-5.
6 Flint R, Halbmeijer N, Meesters N, van Rosmalen J, Reiss I, van Dijk M, et al. Retrospective study shows that doxapram therapy avoided the need for endotracheal intubation in most premature neonates. Acta Paediatr. 2017;106(5):733-9.

7 Vliegenthart RJ, Ten Hove CH, Onland W, Van Kaam AH. Doxapram treatment for apnea of prematurity: a systematic review. Neonatology. 2017;111(2):162-71.

8 Poets CF, Darraj S, Bohnhorst B. Effect of doxapram on episodes of apnoea, bradycardia and hypoxaemia in preterm infants. Biol Neonate. 1999;76(4):207-13.

9 Flint R, Weteringenvan WV, Völler S, Poppe JA, Koch BC, Grootde R, et al. Big data analyses for continuous evaluation of pharmacotherapy: a proof of principle with doxapram in preterm infants. Curr Pharm Des. 2017 Feb 14;23(38):5919-27.

10 Henderson-Smart DJ, Steer PA. Doxapram treatment for apnea in preterm infants (Review). 2013;(4):4-6.
11 Ten Hove $\mathrm{CH}$, Vliegenthart RJ, Te Pas AB, Brouwer E, Rijken M, Van Wassenaer-Leemhuis AG, et al. Long-term neurodevelopmental outcome after doxapram for apnea of prematurity. Neonatology. 2016;110(1):21-6.

12 De Cock RF, Piana C, Krekels EHJ, Danhof M, Allegaert K, Knibbe CAJ. The role of population PK-PD modelling in paediatric clinical research. Eur J Clin Pharmacol. 2011; 67(Suppl 1):S5-16.

13 Leroux S, Turner MA, Guellec CB, Hill H, van den Anker JN, Kearns GL, et al. Pharmacokinetic studies in neonates: the utility of an opportunistic sampling design. Clin Pharmacokinet. 2015;54(12): 1273-85.

14 Falcão AC, Fernández de Gatta MM, Delgado Iribarnegaray MF, Santos Buelga D, García MJ, Dominguez-Gil A, et al. Population pharmacokinetics of caffeine in premature neonates. Eur J Clin Pharmacol. 1997;52(3):2117.

Engbers/Völler/Poets/Knibbe/Reiss/ Koch/Flint/Simons 
15 Koch G, Datta AN, Jost K, Schulzke SM, van den Anker J, Pfister M. Caffeine citrate dosing adjustments to assure stable caffeine concentrations in preterm neonates. J Pediatr. 2017; 191:50-6.

16 Völler S, Flint RB, Beggah F, Reiss I, Andriessen P, Zimmermann LJI, et al. Recently registered midazolam doses for preterm neonates do not lead to equal exposure: a population pharmacokinetic model. J Clin Pharmacol. 2019;59(10):1300-8.

17 Flint R, Simons S, Andriessen P, Liem K, Degraeuwe P, Reiss I, et al. The bioavailability and maturing clearance of doxapram in preterm infants. Arch Dis Child. 2019;104(6):1.

18 Poppe JA, Van Weteringen W, Voller S, Willemsen SP, Goos TG, Reiss IKM, et al. Use of continuous physiological monitor data to evaluate doxapram therapy in preterm infants. Neonatology. 2020;117(4):438-45.

19 Van Den Anker JN. New ways to measure the effect of pharmacotherapy in newborn infants: feasible or not that much? Neonatology. 2020;117(4):436-7.

20 Thomson AH, Kerr S, Wright S. Population pharmacokinetics of caffeine in neonates and young infants. Ther Drug Monit. 1996;18(3): 245-53.

21 Lee TC, Charles B, Steer P, Flenady V, Shearman A. Population pharmacokinetics of intravenous caffeine in neonates with apnea of prematurity. Clin Pharmacol Ther. 1997;61(6):628-40.

22 Charles BG, Townsend SR, Steer PA, Flenady VJ, Gray PH, Shearman A. Caffeine citrate treatment for extremely premature infants with apnea: population pharmacokinetics, absolute bioavailability, and implications for therapeutic drug monitoring. Ther Drug Monit. 2008;30(6):709-16.

23 Shearman P, Mulla H, Kairamkonda V, Spooner N, Gade S, Della Pasqua O, et al. Dried blood spots and sparse sampling: a practical approach to estimating pharmacokinetic parameters of caffeine in preterm infants. Br J Clin Pharmacol. 2013;75(3):80513.

24 Flint RB, Simons SHP, Andriessen P, Liem KD, Degraeuwe PLJ, Reiss IKM, et al. The bioavailability and maturing clearance of doxapram in preterm infants. Pediatr Res. $2020 \mathrm{Jul}$ 22. 